\title{
Saúde bucal na Estratégia de Saúde da Família em um colegiado gestor regional do estado do Piauí
}

\author{
Oral health in the family health strategy \\ in a regional management territory of the state of Piaui
}

Marcoeli Silva de Moura ${ }^{1}$

Flávia Ennes Fonseca Dourado Ferro ${ }^{1}$

Naiana Lustosa da Cunha ${ }^{1}$

Otacílio Batista de Sousa Nétto ${ }^{1}$

Marina de Deus Moura de Lima ${ }^{1}$

Lúcia de Fátima Almeida de Deus Moura ${ }^{1}$

${ }^{1}$ Departamento de Patologia e Clínica Odontológica, Universidade Federal do Piauí. Rua Estudante Danilo Romero 1776, Horto Florestal. 64052-510 Teresina PI. marcoeli-moura@ uol.com.br

\begin{abstract}
The objective of this cross-sectional study was to assess the professional profile of dental-surgeons (DS) who work in the Family Health Strategy (FHS), and on the basis of this reflect upon aspects of the development of dental actions in primary health care in municipalities of a health region of the state of Piaui (Brazil) The study covered the nineteen municipalities of the region. The sample consisted of 111 dentists and data was collected using a questionnaire from August to December 2008. An Excel Ò database was created for the statistical analysis. The dentists were predominantly female, aged 20-39 years, with one to five years of professional experience. Most of them are specialists in a clinical field of dentistry and make home care visits. However, these visits are infrequent and without a health team and they rarely meet with the health team to compare notes. The results indicate that the problems that were detected are likely to be the same in other regions of the country. The lack of an interdisciplinary professional approach among DS is an issue to be properly addressed when examining the role of dentistry and its potentially unquestionable participation in the family health strategy.

Key words Oral health, Family Health Program, Unified Health System, Dental Health Services, Health Policy
\end{abstract}

Resumo Este estudo transversal avaliou o perfil dos cirurgiões-dentistas (CD) que trabalham na Estratégia Saúde da Família (ESF) e a partir deste, refletiu sobre aspectos do desenvolvimento das ações odontológicas na atenção primária em saúde, de municípios de um colegiado do processo de regionalização sanitária do estado do Piauí, Brasil. O estudo abrangeu os dezenove municípios do referido colegiado. No período de agosto a dezembro de 2008, foram aplicados questionários a 111 $C D$, que aceitaram participar do estudo e trabalhavam no respectivo território. Os dados foram transferidos para o programa Excel $\AA$, tabuladose analisados. Os CD avaliados foram predominantemente do gênero feminino, com idade entre 2039 anos, tempo de formado entre um a cinco anos. Eles possuem, em sua maioria, curso de especialização em área clínica. A maioria faz visitas domiciliares, mas sem a presença da equipe, com baixa periodicidade e raramente reúne-se com esta. Os resultados apontam problemas que provavelmente são encontrados em outras realidades do território nacional. A tendência do CD à não interdisciplinaridade é questão ainda em aberto, quando examinado o papel da odontologia e sua potencialmente inquestionável participação na estratégia saúde da família.

Palavras-Chave Saúde bucal, Programa Saúde da Família, Sistema Único de Saúde, Serviços de saúde bucal, Política de saúde 


\section{Introdução}

O Ministério da Saúde brasileiro desde 1994 adotou a atenção primária (básica) em saúde, como estratégia de reorientação do modelo assistencial desenvolvido no SUS, buscando avançar na implantação de ações e serviços, na melhoria dos indicadores de saúde, contribuindo com a qualidade de vida da população assistida. No programa, hoje estratégia saúde da família, que teve como precursor o programa de agentes comunitários de saúde (PACS), existente desde 1991, a odontologia foi oficialmente inserida apenas seis anos depois, em $2000^{1}$.

O incentivo proposto pelo Governo Federal e aceito majoritariamente pelos municípios brasileiros para estimular a incorporação da odontologia na Estratégia Saúde da Família $(E S F)^{2}$; a posterior publicação das Diretrizes da Política Nacional de Saúde Bucal - Brasil Sorridente (2004) e o paralelo amadurecimento acadêmico, técnico e político do movimento sanitário bucal brasileiro têm sido os grandes acontecimentos catalisadores das mudanças em termos de modelo assistencial, de processo de trabalho e da contribuição da saúde bucal coletiva frente ao cenário sanitário brasileiro ${ }^{3,4}$.

Simultaneamente, todo este processo vem resultando na exponencial incorporação de trabalhadores de saúde bucal no SUS, particularmente na atenção básica, através da habilitação de equipes modalidade I (Cirurgião-Dentista CD + Auxiliar de Saúde Bucal ASB) e modalidade II (CD+ASB+ Técnico de Saúde Bucal TSB). Em abril de 2011, já eram 20.640 equipes em 4.829 municípios, e com a expansão prevista no Projeto Mais Saúde do Governo Federal (PAC da Saúde), há a estimativa que serão implantadas até o final de 2011, mais 7.500 novas equipes do Programa Brasil Sorridente, e abertos 400 novos Centros de Especialidades Odontológicas $(\mathrm{CEO})^{5,6}$.

No campo da odontologia em saúde coletiva, os desafios estão colocados: tornar passado o histórico de abandono e de falta de compromisso público com a saúde bucal da população; pensar a saúde bucal coletiva tendo como norte os princípios ordenadores da atenção básica (primeiro contato, longitudinalidade, abrangência e coordenação) e reorganizá-la (especialmente por meio da Estratégia Saúde da Família); garantir a ampliação e a qualificação da atenção especializada (através, principalmente, da implantação de CEO e Laboratórios Regionais de Próteses Dentárias-LRPD), a intensificação da viabilização da adição de flúor nas águas dos municípios com estações de tratamento e desenvolver paulatinamente atividades de vigilância e monitoramento, a partir da iniciativa de criação dos centros colaboradores de vigilância em saúde bucal em todas as macrorregiões brasileiras ${ }^{3,4,7}$.

Quando se avalia o cotidiano das equipes de saúde bucal inseridas na estratégia saúde da família e o desempenho das mesmas, vários são os sinais de fragilidade evidenciados, que a curto, médio e longo prazo podem contribuir para avanços tímidos ou até mesmo a falta desses, que possam ser mensurados em processos avaliativos isentos e imparciais, e talvez o mais grave, contribuir para o desencanto dos profissionais e da população com a presença da odontologia na saúde da família e seus potenciais impactos ${ }^{8-12}$.

Por um lado, a precarização do vínculo trabalhista muitas vezes mediado por contratos ou concursos com seriedade bastante discutível; a elevada rotatividade dos profissionais pelas equipes nos diferentes municípios brasileiros; os inaceitáveis e ilegais, mas reais, duplos vínculos do mesmo profissional em equipes distintas, em municípios distintos. Paralelamente, o descumprimento sistemático por gestores e profissionais das portarias ministeriais que orientam a implantação da estratégia (especialmente das cargas horárias a serem trabalhadas, e da garantia de infraestrutura mínima para o desenvolvimento das atividades clínicas). E por fim, o desconhecimento formal e atitudinal do papel da saúde bucal na atenção básica em saúde, contribuindo decisivamente para isso, a falta de uma formação acadêmica que valorize e oportunize conhecimentos, atitudes e práticas sobre diversos campos da saúde coletiva, são problemas que comprometem sobremaneira a saúde bucal na ESF.

Uma clínica de saúde bucal coletiva e individual que trabalhe numa perspectiva ampliada, com projetos terapêuticos singulares, passando não apenas pela epidemiologia, planejamento e programação, monitoramento e avaliação de serviços de saúde, mas também pela questão do acolhimento, da humanização, da escuta qualificada e do vínculo ${ }^{13}$. Todas estas questões e suas intercessões têm decisiva importância no modo como os profissionais realizam o papel que consideram adequado dentro da estratégia saúde da família, ainda e infelizmente com a priorização hegemônica do atendimento clínico cirúrgicorestaurador-mutilador.

A desvalorização das categorias profissionais ligadas a este núcleo específico da saúde (odontologia) quer nos seus valores simbólicos e nos concretos (salários desestimulantes, ausência de 
efetivas propostas de educação permanente, exclusão do CD e dos profissionais da saúde bucal de nível médio, das capacitações existentes para os outros profissionais nos serviços de saúde, e a própria tendência ao (auto) isolamento da odontologia dentro da equipe multiprofissional) são aspectos que não passam despercebidos e agravam as questões acima levantadas.

Tendo em vista a contextualização anteriormente exposta, este trabalho teve por objetivo analisar o perfil de atuação dos cirurgiões-dentistas que trabalham na estratégia saúde da família e a partir deste, refletir sobre alguns aspectos do desenvolvimento das ações de saúde bucal em municípios de pequeno e médio porte de um colegiado do processo de regionalização da saúde no Estado do Piauí.

\section{Métodos}

O delineamento do estudo foi do tipo observacional, descritivo, transversal sobre a saúde bucal na estratégia saúde da família de todos os municípios de um colegiado gestor regional do Estado do Piauí, sorteado dentre os 11 existentes na versão atual do Plano Diretor de Regionalização da Assistência à Saúde do Estado. O colegiado referido é composto por municípios de pequeno porte (menos de 100 mil habitantes $-\mathrm{n}=18$ ) e médio porte (acima de 100 mil habitantes $-\mathrm{n}=1$ ), classificados de acordo com o censo de 2007 (contagem populacional em 2007) ${ }^{14}$ e o ponto de corte estabelecido pelo Ministério da Saúde para a inclusão dos municípios no Projeto de Expansão e Consolidação da Saúde da Família (PROESF). A realização da pesquisa foi aprovada no Comitê de Ética em Pesquisa da Universidade Federal do Piauí. A presente pesquisa não recebeu financiamento para sua realização.

A intenção inicial seria a realização de um inquérito censitário com todos os cirurgiões dentistas (165) cadastrados nas equipes dos referidos municípios, mas deste total universo, 111 (67,3\%) assinaram o TCLE, após terem recebido todas as informações pertinentes à pesquisa, obedecendo as normas do Conselho Nacional de Saúde para pesquisas com seres humanos. O estudo foi realizado no período de agosto a dezembro de 2008 e analisou individualmente cada CD responsável por sua respectiva Equipe de Saúde Bucal (ESB), que recebeu por sua vez, todas as informações pertinentes à pesquisa, e os que concordaram em participar, assinaram o Termo de Consentimento Livre e Esclarecido (TCLE).
A estrutura do questionário foi baseada em pesquisas da literatura e adaptada aos objetivos do estudo. Foi realizado inicialmente um pré-teste com dez cirurgiões-dentistas que não participaram do estudo para adequação do instrumento de coleta de dados utilizado, aferição das dificuldades encontradas e capacitação dos pesquisadores envolvidos no projeto com a finalidade de verificar o entendimento em relação às perguntas fechadas do questionário, conservando ou alterando-as, como forma de adequação. Foram avaliados aspectos relativos ao perfil dos cirurgiões-dentistas, à natureza das atividades clínicas e extraclínicas realizadas, rotina de trabalho, por meio de questionário semiestruturado, que continha perguntas fechadas e abertas.

A metodologia utilizada para analisar os dados obtidos nas respostas dos questionários foi a de um estudo quantitativo com tratamento estatístico descritivo, utilizando-se tabelas, produzidas a partir de planilhas processadas pelo programa Microsoft Excel (Microsoft), para representar as ocorrências dos resultados percentualmente.

\section{Resultados}

A Tabela 1 apresenta o perfil dos CD que trabalham na ESF no estado do Piauí, na qual se observa que estes são predominantemente do gênero feminino, com idade entre 20-39 anos, tempo de formado entre um a cinco anos, em universidade pública. Eles possuem, em sua maioria, curso de pós-graduação, do tipo especialização, em área clínica.

A Tabela 2 apresenta o processo de trabalho da equipe de saúde bucal na ESF, na qual se destaca que apenas $28 \%$ dos CD trabalham os cinco dias da semana, $48,6 \%$ residem na cidade onde trabalham e 46,9\% reúnem-se regularmente com a equipe de saúde da família.

A Tabela 3 apresenta as atividades realizadas pela equipe de saúde bucal na ESF, na qual se observa que apenas $27 \%$ dos CD realizam visita domiciliar com a equipe e em $48,6 \%$ dos casos toda a população é visitada independentemente de sua necessidade.

\section{Discussão}

A inserção da odontologia na ESF tem representado uma dualidade integradora: de um lado a possibilidade concreta de vivência das ações e ser- 
viços públicos de saúde, a partir de um campo de saberes comuns às diversas profissões do setor saúde, de outro, o desenvolvimento de um núcleo específico de atuação da saúde bucal. Estes

Tabela 1. Perfil dos cirurgiões-dentistas que integram as equipes de saúde bucal na Estratégia Saúde da Família,

\begin{tabular}{|c|c|c|}
\hline Característica & Frequência & $\%$ \\
\hline \multicolumn{3}{|l|}{ Gênero } \\
\hline Masculino & 49 & 44,5 \\
\hline Feminino & 62 & 55,5 \\
\hline \multicolumn{3}{|l|}{ Idade } \\
\hline 20-29 anos & 42 & 37,8 \\
\hline 30-39 anos & 39 & 5,2 \\
\hline 40-49 anos & 17 & 15,3 \\
\hline mais de 50 anos & 13 & 11,7 \\
\hline \multicolumn{3}{|l|}{ Tempo de Formado } \\
\hline$>$ de 1 ano & 8 & 7,2 \\
\hline 1 a 5 anos & 40 & 36,1 \\
\hline 6 a 10 anos & 27 & 24,3 \\
\hline acima de 10 anos & 36 & 32,4 \\
\hline \multicolumn{3}{|l|}{ Faculdade em que se formou } \\
\hline Pública & 85 & 76,6 \\
\hline Particular & 23 & 20,7 \\
\hline Sem Resposta & 3 & 2,7 \\
\hline \multicolumn{3}{|c|}{ Possui Curso de Pós-Graduação } \\
\hline Sim & 90 & 81,0 \\
\hline Não & 21 & 19,0 \\
\hline \multicolumn{3}{|c|}{ Tipo de Curso de Pós-graduação } \\
\hline Não Possui & 21 & 19,0 \\
\hline Aperfeiçoamento & 29 & 26,0 \\
\hline Especialização & 60 & 54,0 \\
\hline Mestrado & 1 & 1,0 \\
\hline \multicolumn{3}{|c|}{ Área do Curso de Pós-graduação } \\
\hline Não possui & 21 & 19,0 \\
\hline Saúde Pública & 31 & 27,8 \\
\hline Clínica & 41 & 37,0 \\
\hline Saúde Pública e Clínica & 18 & 16,2 \\
\hline \multicolumn{3}{|l|}{ Tempo de Trabalho no PSF } \\
\hline$>1$ ano & 13 & 11,7 \\
\hline $1-4$ anos & 56 & 50,5 \\
\hline 5-7 anos & 42 & 37,8 \\
\hline \multicolumn{3}{|l|}{ Já trabalhou em outro PSF } \\
\hline Sim & 60 & 54,0 \\
\hline Não & 51 & 46,0 \\
\hline \multicolumn{3}{|l|}{ Quanto tempo no outro PSF } \\
\hline Não trabalhou & 51 & 46,0 \\
\hline$>1$ ano & 10 & 9,03 \\
\hline $1-4$ anos & 43 & 38,7 \\
\hline 5-7 anos & 7 & 6,3 \\
\hline \multicolumn{3}{|c|}{ Está satisfeito com sua remuneração? } \\
\hline Sim & 11 & 9,9 \\
\hline Não & 100 & 90,1 \\
\hline \multicolumn{3}{|c|}{ Como foi efetivada a contratação? } \\
\hline Concurso Público & 13 & 11,7 \\
\hline Outro & 98 & 88,3 \\
\hline
\end{tabular}

lados podem e devem dialogar mediadas por um processo de trabalho pautado em relações multiprofissionais, em territorialização, em responsabilidade sanitária sobre uma população adscrita $^{15}$. Dessa forma, o cuidado em saúde bucal passou a exigir o delineamento de uma equipe de trabalho que se relacione com os usuários e que

Tabela 2. Processo de Trabalho da equipe de saúde bucal na Estratégia Saúde da Família em municípios piauienses, de pequeno e médio porte populacional, Teresina, 2011.

\begin{tabular}{lrr}
\hline \multicolumn{1}{c}{ Questionamento } & Frequência & \% \\
\hline $\begin{array}{l}\text { Quantos dias da semana trabalha } \\
\text { na ESF? }\end{array}$ & & \\
2 & 9 & 8,1 \\
3 & 47 & 21,6 \\
4 & 31 & 42,3 \\
5 & & \\
Distância da cidade de origem para & & \\
a cidade que trabalha & 54 & 48,6 \\
Mora na cidade & 30 & 27,0 \\
50 a $100 \mathrm{Km}$ & 9 & 8,1 \\
101 a $200 \mathrm{Km}$ & 13 & 11,7 \\
201 a $300 \mathrm{Km}$ & 4 & 3,6 \\
301 a $400 \mathrm{~km}$ & 1 & 1,0 \\
acima $400 \mathrm{Km}$ &
\end{tabular}

Recebeu algum tipo de capacitação para o PSF?

$\begin{array}{lll}\text { Sim } & 71 & 64,0\end{array}$

Acha-se capacitado para atuar no PSF?

Sim $93 \quad 83,8$

Parcialmente $\quad 16 \quad 14,4$

$\begin{array}{lll}\text { Não } & 2 & 1,8\end{array}$

A capacitação foi suficiente?

Não foi capacitado $\quad 40 \quad 36,0$

Sim $32 \quad 28,9$

Parcialmente $\quad 34 \quad 30,6$

Não $\quad 5 \quad 4,5$

Reúne-se com a equipe de saúde?
$\quad$ Sim

Não $\quad 14 \quad 12,6$

Às vezes $\quad 45 \quad 40,5$

Qual a periodicidade da reunião com a equipe?

Semanal $15 \quad 13,5$

Quinzenal $13 \quad 11,7$

Mensal $\quad 35 \quad 31,5$

Raramente $34 \quad 30,7$

Não se reúne $\quad 14 \quad 12,6$

"Os resultados ultrapassam 100\% porque era permitido marcar mais de uma alternativa. 
participe da cogestão dos serviços, buscando identificar e responder as demandas da população e a ampliação do acesso desta às ações de promoção, prevenção e recuperação da saúde bucal, por

Tabela 3. Atividades realizadas pela equipe de saúde bucal da Estratégia Saúde da Família em municípios piauienses de pequeno e médio porte populacional, Teresina, 2011.

\begin{tabular}{|c|c|c|}
\hline Característica & Frequência & $\%$ \\
\hline \multicolumn{3}{|l|}{$\begin{array}{l}\text { Quais ações realiza em } \\
\text { ambulatório?*}\end{array}$} \\
\hline Dentística & 110 & 99,1 \\
\hline Exodontia & 105 & 94,6 \\
\hline $\begin{array}{l}\text { Prevenção (Profilaxia, } \\
\text { Instrução de higiene, ATF) }\end{array}$ & 99 & 89,2 \\
\hline Periodontia Básica & 78 & 70,3 \\
\hline Urgência & 20 & 18,2 \\
\hline \multicolumn{3}{|l|}{$\begin{array}{l}\text { Visita Domiciliar conjunta com a } \\
\text { equipe? }\end{array}$} \\
\hline Sim & 30 & 27,0 \\
\hline Não & 33 & 29,7 \\
\hline Às vezes & 22 & 19,9 \\
\hline Não realiza visita & 26 & 23,4 \\
\hline \multicolumn{3}{|l|}{ Qual a periodicidade da visita? } \\
\hline Semanal & 29 & 26,1 \\
\hline Quinzenal & 14 & 12,7 \\
\hline Mensal & 16 & 14,4 \\
\hline Raramente & 26 & 23,4 \\
\hline Não realiza visita & 26 & 23,4 \\
\hline \multicolumn{3}{|l|}{$\begin{array}{l}\text { Quem seleciona os locais a serem } \\
\text { visitados? }\end{array}$} \\
\hline $\begin{array}{l}\text { Agente Comunitário de Saúde } \\
\text { (ACS) }\end{array}$ & 53 & 47,7 \\
\hline Equipe & 21 & 19,0 \\
\hline Cirurgião-dentista & 6 & 5,4 \\
\hline Outro & 5 & 4,5 \\
\hline Não realiza visita & 26 & 23,4 \\
\hline \multicolumn{3}{|l|}{$\begin{array}{l}\text { Que indivíduos/grupos são } \\
\text { visitados? }\end{array}$} \\
\hline Todos & 54 & 48,6 \\
\hline $\begin{array}{l}\text { Gestantes, Recém-nascidos, } \\
\text { Diabéticos/Hipertensos, } \\
\text { Acamados }\end{array}$ & 31 & 28,0 \\
\hline Não realiza visita & 26 & 23,4 \\
\hline \multicolumn{3}{|l|}{$\begin{array}{l}\text { Ações realizadas nas visitas } \\
\text { domiciliares?* }\end{array}$} \\
\hline Educação em saúde bucal & 80 & 72,1 \\
\hline Aplicação Tópica de Flúor & 42 & 37,8 \\
\hline $\begin{array}{l}\text { Escovação supervisionada/ } \\
\text { limpeza boca bebê }\end{array}$ & 36 & 32,4 \\
\hline Exame Clínico & 30 & 27,0 \\
\hline Levantamento Epidemiológico & 4 & 3,6 \\
\hline Não realiza visita & 26 & 23,4 \\
\hline
\end{tabular}

Os resultados ultrapassam $100 \%$ porque era permitido marcar mais de uma alternativa. meio de medidas de caráter individual e coletivo e mediante o estabelecimento de vínculo ${ }^{4}$.

O Estado do Piauí possui 224 municípios e uma população estimada de 3.032.421 habitantes ${ }^{14}$. Atualmente no Estado encontram-se instaladas 1.105 Equipes de Saúde da Família (ESF) e 942 Equipes de Saúde Bucal (ESB) [933 modalidade I ( $\mathrm{CD}$ e ASB) e nove modalidade II (CD, ASB e TSB)]. A razão entre Equipes de Saúde Família/Equipes de Saúde Bucal é de 1:0,85, o que evidencia a existência de 163 ESF sem ESB no Estado, comportamento também observado nos dados brasileiros gerais, caracterizando o descumprimento da Portaria Ministerial 673 de 03/ 06/2003, que atualizou e reviu o incentivo financeiro às ações de saúde bucal, no âmbito da ESF, como parte integrante do Piso de Atenção Básica (PAB), e estabelecia que poderiam ser implantadas, nos municípios, quantas equipes de saúde bucal fossem necessárias, a critério do gestor municipal, desde que não ultrapassassem o número existente de equipes de saúde da família, na proporção de 1:1 com ação junto as ESF (art.1), ou seja equiparava o número de $\mathrm{ESF} / \mathrm{ESB}^{16}$.

O Piauí, por apresentar um elevado número de municípios de pequeno porte $(73,67 \%$ dos municípios $<10$ mil habitantes) ${ }^{14}$ consegue atingir altas coberturas populacionais em termos de atenção básica com um número menor de equipes, às vezes com uma única equipe por município, e a implantação destas equipes significa uma concreta expansão de serviços básicos e de infraestrutura sanitária que outrora sequer existiam. Equipes únicas são polissêmicas: podem ser de fato a porta de entrada no SUS ou uma porta que embora aberta, permanece permanentemente fechada para o cuidado em saúde. Entretanto se desconhece de que forma concreta os princípios do SUS e da estratégia saúde da família são aplicados e vivenciados nesta realidade peculiar.

A partir de uma leitura acadêmica e política da realidade sanitária piauiense, especula-se que grande parte da aceleração do processo de habilitação de equipes de saúde da família e de equipes de saúde bucal tenha visado no Estado do Piauí e provavelmente em outros estados brasileiros, primariamente, o recebimento dos recursos tidos como incentivos do Ministério da Saúde, nem sempre utilizados de forma devida para a implantação das equipes, para adequação e manutenção da infraestrutura mínima necessária, para oferecimento de salários mais interessantes para a adesão e vinculação dos profissionais à estratégia e aos processos de trabalho propostos. 
Pondera-se que em relação aos recursos que compõem o bloco do financiamento da atenção básica via modalidade de transferência fundo-afundo (Fundo Nacional de Saúde-Fundo Municipal de Saúde) segundo o Pacto pela Saúde, pouco se tem clareza sobre a verdadeira destinação dos mesmos, especialmente nos menores municípios brasileiros, onde as estruturas de controle social do SUS são incipientes e atreladas aos gestores, como também são as câmaras municipais, ao passo que representantes do Ministério Público, sobrecarregados com outras demandas, não dão às questões da saúde a atenção e a prioridade devidas ${ }^{17,18}$.

Avaliando-se o perfil dos CD estudados ( $\mathrm{Ta}$ bela 1), confirmam-se as estatísticas encontradas no Perfil Atual e Tendências do Cirurgião Dentista Brasileiro, publicado em 2010, com dados referentes a 2008, que apontam o Piauí com $53 \%$ da população de CD do sexo feminino, sendo esta uma tendência nacional que é observada desde o final dos anos $90^{19}$. Em relação à faixa etária predominante entre os $\mathrm{CD}$, novamente há concordância com os dados de Morita et al. ${ }^{19}$ nos quais a maior concentração de profissionais se encontra na faixa etária de 26 a 35 anos. $\mathrm{Na}$ amostra estudada também predomina profissionais com menor tempo de exercício na profissão. Com o aumento de cursos de odontologia no país de acordo com o Censo da Educação Superior de 2008 (197 cursos), verificado especialmente a partir da segunda metade da década de 1990, houve um aumento substancial do número de profissionais com menos de 10 anos de formado ${ }^{20}$. Em direção oposta observou-se também um número expressivo de profissionais que se formaram há mais de 10 anos. Isso demonstra que nas equipes de saúde bucal da estratégia saúde da família piauiense, encontra-se uma heterogeneidade de profissionais no tocante à experiência profissional, o que pode gerar dependendo dos processos de trabalho adotados nos municípios, um clima de cooperação mútua ou um verdadeiro conflito de gerações, conhecimentos, atitudes e práticas.

A graduação dos profissionais aconteceu hegemonicamente em faculdades públicas e apesar do pouco tempo decorrido da graduação, os CD em questão já passaram por cursos de pós-graduação: aperfeiçoamento/especialização, especialmente em áreas clínicas. $\mathrm{O}$ acesso maciço à pósgraduação pode ser decorrente, do incentivo/abertura que o Conselho Federal de Odontologia proporcionou a diversas instituições, para que juntamente com as Universidades e Associações de classe pudessem abrir cursos de pós-graduação no Brasil dos anos 1990 e na primeira década do século XXI. Quando estes dados são comparados a outra realidade dentro do mesmo Estado, particularmente em sua capital Teresina, Vilarinho et al. $2006^{21}$, encontraram resultados distintos, somente $32,7 \%$ dos cirurgiões-dentistas possuíam pós-graduação, e apresentavam entre 17 e 23 anos de formado. A explicação para essa diferença é que a gestão da Fundação Municipal de Saúde de Teresina, ao decidir técnica e politicamente pela implantação da saúde bucal na ESF em 2003, convidou os dentistas que já trabalhavam em ambulatórios dos serviços de saúde bucal municipal para participar do programa em construção justificando a diferença do perfil dos CD que trabalham na capital e interior do Piauí.

A adoção da estratégia saúde da família como mercado de trabalho é recente, e para $62,2 \%$ da população de profissionais estudada, não é superior a quatro anos de vínculo na atual equipe, ainda que $54 \%$ dos estudados já tenham passado por uma experiência anterior de trabalho em outra equipe de saúde da família. A experiência em duas ou mais equipes de saúde da família pode de alguma forma estar relacionada à elevada rotatividade de profissionais entre as equipes, provavelmente devido à procura de condições de trabalho menos precárias, à instabilidade profissional, decorrente de vínculos empregatícios instáveis, ao interesse gradativamente crescente por trabalhar em municípios maiores, mais bem estruturados e mais próximos da capital, entre outros ${ }^{18}$. Mesmo na experiência anterior de vínculo com a saúde da família, o tempo de trabalho não excedeu quatro anos para $43 \%$ dos examinados. Esse dado torna-se compreensível quando se resgata o fato de que a inserção da odontologia na saúde da família só aconteceu em dezembro do ano 2000, e em 2001, segundo relatos do próprio Ministério da Saúde, havia apenas 200 equipes de saúde bucal implantadas no Brasil.

O profissional de saúde bucal inserido na estratégia saúde da família deveria estar preparado e conhecer em tese como conduzir a equipe de saúde bucal, parte indissolúvel da equipe de saúde da família, para atingir os objetivos pensados pela estratégia. Quando se observa o maior interesse dos $\mathrm{CD}$ em pós-graduações na área clínica, isto resulta provavelmente da força imagética que essas disciplinas, tidas como profissionalizantes, têm na formação de graduação em odontologia e no ideário simbólico da profissão, secundarizando ou marginalizando as questões inerentes 
à saúde coletiva. A existência de matrizes curriculares orientadas para uma formação mais técnica, com uma prática clínica individualizada, fragmentada, biocêntrica, curativa, com ênfase no uso de tecnologias de ponta ${ }^{22}$ continua sendo a tônica dominante, apesar da retórica recente e crescente de aproximação com o SUS, provocada especialmente pelas Novas Diretrizes Curriculares Nacionais para os Cursos da área de saúde, e por importantes iniciativas interministeriais voltadas para a formação de graduação em saúde, como PET Saúde e o Pró-Saúde, este último no qual o curso de odontologia da UFPI está inserido desde $2005^{23,24}$.

O não cumprimento da carga horária pelo profissional nas equipes em relação ao preconizado pelas portarias ministeriais condensadas na Política Nacional da Atenção Básica (PNAB) ${ }^{17}$, (os cirurgiões-dentistas avaliados, em sua maioria, trabalham quatro dias na ESF), possivelmente está associado ao fato de que $51,4 \%$ dos dentistas não moram no município onde trabalham, o que pode se constituir em fator limitante para o trabalho (Tabela 2). A dificuldade de interiorização do trabalhador em saúde no Brasil que prefere se concentrar nas regiões mais desenvolvidas, nas capitais, nos municípios de maior porte é uma realidade e tem sido uma das grandes dificuldades da expansão da estratégia saúde da família. Nos municípios com até 20 mil habitantes é onde mais intensamente se sente a ausência do CD permanentemente residente e possivelmente nestes municípios encontram-se as implantações mais vulneráveis de equipes de saúde bucal na ESF.

Ainda em relação a esta questão, somente $28 \%$ dos entrevistados trabalham os cinco dias da semana (Tabela 2), um dado claramente contrário à PNAB. O estabelecimento dessa carga horária para os profissionais das equipes de saúde sustenta-se na ideia de que essa é uma condição necessária para a criação de vínculo e o estabelecimento do acolhimento entre os membros da equipe e entre estes e a população. E, ainda, hipoteticamente, para um melhor desenvolvimento do processo de trabalho das equipes, tanto do ponto de vista gerencial e organizacional, quanto assistencial. Especula-se que acordos internos com os gestores locais, fragilidade nas supervisões estaduais e a necessidade de manutenção das equipes funcionando ainda que precariamente, de maneira distante da ideal, são fatores que contribuem para que ainda persistam graves e crônicas distorções no cumprimento da carga horária a ser trabalhada. Questões ligadas à insatisfação com a remuneração recebida, vínculos trabalhistas instáveis, apenas 11,7\% dos CD avaliados foram contratados via concurso público e a apontada necessidade de trabalhar em mais de um emprego em mais de uma cidade, para garantir uma renda adequada, são prováveis explicações complementares para este fenômeno (Tabela 1). Não trabalhando o tempo devido, fica fácil perceber que o processo e a natureza do trabalho desenvolvido ficam comprometidos. O gestor tergiversa, o profissional talvez se angustia, a população com certeza sofre de pouca assistência, de má assistência, de não assistência e de muito mais.

Em relação à percepção de competências necessárias ao trabalho com a saúde da família, há resultados controversos, uma vez que $83,8 \%$ dos profissionais sentem-se aptos ao trabalho, ao passo que o número de profissionais que passaram por algum processo de capacitação específica para atuar no programa cai para $64 \%$ e destes apenas $28,9 \%$ considerem-na suficiente para trabalhar conceitos relativos aos princípios ordenadores da atenção básica, fundamentos de organização do campo da saúde bucal na atenção básica (planejamento, monitoramento e avaliação, indicadores de saúde bucal, processo de trabalho em equipe, organização da demanda, etc.).

Refletindo sobre a questão da capacitação para o trabalho no PSF, é necessário questionar onde aconteceu, se é que aconteceu, esse momento educativo. Será que os cursos de graduação já contemplam de fato e concretamente a ESF nos seus currículos? E se o fazem como fazem? Tendo em vista que a maioria dos $C D$ analisados tem menos de dez anos de formado, podem já ter recebido ou não a influência mais ou menos intensa da adoção das novas diretrizes curriculares dos cursos da área da saúde, e sua orientação clara para uma formação acadêmica que contemple o SUS em seus múltiplos espaços de produção de saúde ou doença. A intensidade com que cresceram nos últimos anos, as pós-graduações na área de saúde coletiva, algumas financiadas pelo próprio Ministério da Saúde, outras privadas, foi capaz de preparar os profissionais recém-formados? Havendo capacitação em qualquer tempo/espaço, talvez esta não tenha sido suficiente para desenvolver com tranquilidade, $o$ conhecimento, as habilidades e as competências para o trabalho a ser desempenhado. Tendo o processo educativo um caráter permanente e contínuo, como olhar para as políticas de educação continuada no Sistema Único de Saúde? Talvez, ainda mais preocupante é a situação dos 36\% da amostra estudada que não receberam qualquer 
capacitação, perdendo-se de certa forma, a oportunidade do profissional de já começar trabalhando de forma diferenciada e adequadamente instruída ${ }^{22-26}$.

Por sua vez, a incapacidade e porque não a falta de vontade política das secretarias municipais de saúde e dos colegiados gestores regionais, e também das secretarias estaduais de saúde, e de seus mecanismos via polos permanentes de educação, dos órgãos intergestores (CIB) e do Conselho Estadual de Saúde em colocar em prática e acompanhar regionalmente a Política Nacional de Educação Permanente em Saúde, repercute negativamente na consolidação de uma estratégia que, em tese, é considerada uma prioridade nacional na área da saúde ${ }^{27}$.

Neste estudo, observam-se dados preocupantes com relação à interdisciplinaridade. (Tabela 2). As reuniões da equipe (médico, enfermeiro, auxiliar de enfermagem, cirurgião-dentista, auxiliar de saúde bucal e agente comunitário) deveriam ser realizadas semanalmente, como instrumento de integração interdisciplinar e multiprofissional, de possibilidade de desenvolvimento de projetos terapêuticos amplos, comuns e integrados, e um espaço privilegiado de partilha dentro da equipe, do conhecimento dos problemas da comunidade e de discussão sobre formas mais efetiva de solucioná-los. No formato atual das equipes, os profissionais com menor assiduidade nas reuniões da equipe são provavelmente médicos e CD. A multiprofissionalidade presente na estratégia saúde da família, não garante interdisciplinaridade ou integração dos projetos de intervenção. Pedrosa e Teles estudando a estratégia saúde da família em Teresina ${ }^{28}$ observaram que o pequeno relacionamento interno da equipe revela a inexistência de responsabilidade coletiva pelos resultados do trabalho, levando à descontinuidade entre as ações específicas de cada profissional, observando-se desarticulação entre as ações curativas, educativas e administrativas, bem como um baixo grau de interação entre os membros da equipe.

Em relação às atividades desenvolvidas pelos cirurgiões-dentistas na ESF, há ainda uma elevada carga de necessidades ligadas a ações de assistência odontológica clínica individual, sobressaindo-se os procedimentos restauradores, periodontais e cirúrgicos (exodontias), provocados pela negligência histórica de atendimento odontológico público e universal, bem como pelo crônico descompasso entre o trabalho realizado pelas equipes de saúde e o necessário para proporcionar uma maior cobertura populacional.
Importante ressaltar ainda a relevante concentração de atividades preventivas individuais e coletivas (profilaxia, higiene bucal supervisionada, educação em saúde bucal, aplicações tópicas de flúor), que representa potencialmente um avanço qualitativo na natureza do trabalho realizado, menores custos, ainda que se considere a natureza restritiva, excessivamente biológica e limitada dos procedimentos exclusivamente preventivos. A consolidação internacional e nacional do movimento de promoção de saúde e a constatação a partir de evidências avaliativas de serviços de saúde produziram um consenso que as equipes de saúde bucal da família deveriam ter como objetivo principal, sem prejuízo das demais atividades, o desenvolvimento de atividades com base na promoção de saúde ${ }^{29}$.

Segundo os dados obtidos neste estudo a visita domiciliar ainda não foi adequadamente incorporada na rotina dos CD. Essa visita tem periodicidade muito variada e os locais selecionados para a mesma são em sua maioria designados pelo agente comunitário de saúde. Vários são os obstáculos para a visita domiciliar conjunta no cotidiano das equipes: a prioridade que cada equipe particularmente confere à atividade; a disponibilidade de veículos para conduzir os profissionais (e o equacionamento de que profissionais devem participar da visita) ou as visitas realizadas em deslocamentos pedestres pelo território da comunidade, nem sempre bem quistos e realizados pelos profissionais. O que se pode constatar a partir desses resultados é que a visita domiciliar é ainda uma atividade pouco executada/explorada pelos $\mathrm{CD}$, e a ela não é dada a devida importância. A incorporação do atendimento domiciliar aponta para uma reestruturação e reorganização das práticas de saúde para além dos muros dos serviços de saúde, deslocando seu olhar para o espaço domicílio das famílias e comunidades, nas quais as práticas de vida acontecem ${ }^{30}$.

Quando as visitas domiciliares acontecem, as atividades mais frequentemente realizadas são as de natureza educativa. Há um leque amplo de significações e realizações do ato educativo nestas respostas. Tradicionalmente, o estado da arte da educação realizada pelos profissionais de saúde tem sido sinônimo de atividades de transmissão - ainda que não intencional - autoritária e normatizadora de conhecimentos, desvinculadas dos modos de andar a vida da população usuária ${ }^{31}$. Propostas emancipatórias de educação popular, que têm como premissa o fato de a educação não ser considerada "o dizer aos homens o que é importante, mas facilitar as condições, para que 
eles mesmos vejam a importância das coisas", e que funcionam como instrumentos de promoção de saúde, não são hegemônicas no cotidiano das equipes ${ }^{32,33}$. As atividades educativas dependendo do modo como são conduzidas podem, pela oportunidade que proporcionam de ativar mudanças, atitudes, hábitos e comportamentos, trazer benefícios que culminam na melhoria da qualidade de vida das pessoas ${ }^{31}$. Segundo Emmi e Barroso ${ }^{34}$, as orientações de higiene oral, o maior acesso ao atendimento odontológico e as visitas domiciliares foram apontados pelos usuários como as melhorias consideradas de maior importância para a população com a implantação do PSB em um distrito do Pará.

À presença da odontologia na estratégia saúde da família talvez possa cunhar-se o mesmo aforismo tantas vezes atrelado ao SUS: 'não se trata de um problema sem solução, mas de uma solução com inúmeros e (in)sondáveis problemas', mais ou menos intensos, na medida do avançar das experiências de construção de sistemas locais de saúde, da valorização da saúde bucal, como algo tangível à sociedade brasileira e indissociável da produção de saúde, que acontece diuturnamente pelas cidades e campos do Brasil, sob

\section{Colaboradores}

MS Moura, FEFD Ferro e NL Cunha trabalharam na concepção, delineamento, análise e interpretação dos dados, redação do artigo e revisão crítica, aprovação da versão a ser publicada, sendo que os dois últimos também coletaram dados, OB de Sousa Nétto, MDM Lima e LFAD Moura participaram da redação do artigo, revisão crítica e aprovação da versão a ser publicada. a responsabilidade sanitária solidária dos gestores, dos profissionais de saúde, prestadores de serviços e também dos usuários destes serviços.

As questões apontadas pelo trabalho realizado com dentistas vinculados a equipes da estratégia saúde da família de um colegiado gestor regional do Estado do Piauí demonstram problemas que provavelmente são encontrados de forma disseminada pelo Brasil. Há a formação profissional ainda distante do desejado quando se trata da aproximação real com o SUS e suas multifacetadas experiências, e também existem as dificuldades da educação permanente dos profissionais já envolvidos na saúde da família. O conhecimento (a falta de) e a vinculação (a falta de) efetiva aos princípios e às práticas da estratégia saúde da família; as prementes questões gerenciais; os processos de trabalho multiprofissional da estratégia em choque com a tendência do $\mathrm{CD}$ ao uniprofissionalismo e à não interdisciplinaridade. Todas estas são dúvidas latentes na segunda década do século XXI, quando examinado o papel da odontologia (a partir dos CD) e sua potencialmente inquestionável participação na estratégia saúde da família.

\section{Referências}

1. Narvai PC, Frazão P. O SUS e a Política Nacional de Saúde Bucal. In: Pereira AC. Tratado de Saúde Coletiva em Odontologia. Nova Odessa: Napoleão Editora; 2009. p. 18-34.

2. Brasil. Ministério da Saúde (MS). Portaria N.o 1.444 de 28 de dezembro de 2000. Estabelece incentivo financeiro para a reorganização da atenção à saúde bucal prestada nos municípios por meio do Programa de Saúde da Família. Diário Oficial da União 2000; dez 29.

3. Brasil. Ministério da Saúde (MS). Secretaria de Atenção à Saúde. Departamento de Atenção Básica. Coordenação Nacional de Saúde Bucal. Diretrizes da Política Nacional de Saúde Bucal. Brasília: MS; 2004.

4. Brasil. Ministério da Saúde (MS). Secretaria de Atenção à Saúde. Departamento de Atenção Básica. Cadernos de Atenção Básica n. 17, Brasília: MS; 2006.

5. Brasil. Ministério da Saúde (MS). Departamento de Atenção Básica. Atenção Básica e Saúde da Família. [acessado 2011 set 19]. Disponível em: http://dab. saude.gov.br/abnumeros.php\#mapas.

6. Brasil. Mais Saúde. Direito de Todos. Pilares. [acessado 2011 set 19]. Disponível em: http://bvsms. saude.gov.br/bvs/pacsaude/estrutura1.php. 
7. Brasil. Ministério da Saúde (MS). Portaria MS/SAS no 939, de 21 de dezembro de 2006. Diário Oficial da União 2006; dez 22.

8. Baldani MHP, Fadel CB, Possamai T, Queiroz MGS A inclusão da odontologia no Programa Saúde da Família no estado do Paraná, Brasil. Cad Saude Publica 2005; 21(4):1026-1035.

9. Araujo YP, Dimenstein M. Estrutura e organização do trabalho do cirurgião-dentista no PSF de municípios do Rio Grande do Norte. Cien Saude Colet 2006; 11(1):219-227.

10. Martelli PJL, Cabral APS, Pimentel FC, Macedo CLSV, Monteiro IS, Silva SF. Análise do modelo de atenção à saúde bucal em municípios do estado de Pernambuco. Cien Saude Colet 2008; 13(5):1669-1674.

11. Pereira CRS, Patrício AAR, Araújo FAC, Lucena EES, Lima KC, Roncalli AG. Impacto da Estratégia Saúde da Família com equipe de saúde bucal sobre a utilização de serviços odontológicos. Cien Saude Colet 2009; 25(5):985-996.

12. Soares FF, Figueiredo CRV, Borges NCM, Jordão RA, Freire MCM. Atuação da equipe de saúde bucal na estratégia saúde da família: análise dos estudos publicados no período 2001-2008. Cien Saude Colet $2011 ; 16(7): 3169-3180$.

13. Campos GWS, Amaral MA. A clínica ampliada e compartilhada, a gestão democrática e redes de atenção como referenciais teórico-operacionais para a reforma do hospital. Cien Saude Colet 2007; 12(4):849-859.

14. Instituto Brasileiro de Geografia e Estatística (IBGE). População recenseada e estimada, segundo os municípios - Piauí - 2007. [acessado 2011 jan 29]. Disponível em: http://www.ibge.gov.br/home/estatistica/ populacao/contagem2007/defaulttab.shtm.

15. Campos GWS. Saúde Pública e Saúde Coletiva: Campo e Núcleo de Saberes e Práticas. Cien Saude Colet 2000; 5(2):219-230.

16. Brasil. Ministério da Saúde (MS). Portaria N. ${ }^{\circ}$ 673/ GM de 3 de junho de 2003 [acessado 2011 set 20]. Disponível em: http://www.atencaoprimaria.to.gov. br/downloads/port673.03_relacao.pdf.

17. Brasil. Ministério da Saúde (MS). Secretaria de Atenção a Saúde. Departamento de Atenção Básica. Política Nacional de Atenção Básica. Brasília: MS; 2006.

18. Sousa MF. Os sinais vermelhos do PSF. São Paulo: Hucitec; 2002.

19. Morita MC, Haddad AE, Araújo ME. Perfil Atual e Tendências do Cirurgião-dentista Brasileiro. Maringá: Dental Press International; 2010.

20. Cabral ED, Caldas Júnior AF, Cabral HAM. Influence of the patient's race on the dentist's decision to extract or retain a decayed tooth. Communit Dent Oral Epidemiol 2005; 33(6):461-466.

21. Vilarinho SMM, Mendes RF, Prado Jr RR. Perfil dos cirurgiões-dentistas integrantes do Programa de Saúde da Família em Teresina (PI). Rev Odonto Ciência 2007; 22(55):48-54.

22. Narvai PC. Recursos humanos para promoção de saúde bucal: Um olhar no início do século XXI. In: Kringer L. Promoção de Saúde Bucal. São Paulo: Artes Médicas; 2003. p. 475-494.
23. Brasil. Ministério da Educação (MEC). Curso de Graduação em Odontologia Proposta de Diretrizes Curriculares [acessado 2011 jul 13]. Disponível em: http://portal.mec.gov.br/cne/arquivos/pdf/Odont. pdf.

24. Brasil. Ministério da Saúde. Ministério da Educação. Programa Nacional de Reorientação da Formação Profissional em Saúde - Pró-Saúde: objetivos, implementação e desenvolvimento potencial. Brasília: MS; 2007.

25. Brasil. Ministério da Saúde (MS). Secretaria de Atenção à Saúde. Departamento da Atenção Básica. Avaliação Normativa do Programa Saúde da Família no Brasil: Monitoramento da implantação e funcionamento das equipes de saúde da família: 2001-2002. Brasília: MS; 2004 (Série C, Projetos, programas relatórios).

26. Medeiros CLA, Queiroz MDD, Sousa GCA. Expectativas de Cirurgiões-dentistas sobre a inserção da saúde bucal no Programa Saúde da Família. Rev Eletrônica Enfermagem 2007; 9(2):379-388.

27. Brasil. Ministério da Saúde. Secretaria de Gestão do Trabalho e da Educação na Saúde. Departamento de Gestão da Educação em Saúde. Política Nacional de Educação Permanente em Saúde. Brasília: MS; 2009.

28. Pedrosa JTS, Teles JBM. Consenso e diferenças em equipes do Programa de Saúde da Família. Rev Saude Publica 2001; 35(2):303-311.

29. Noro LRA. Saúde Bucal na Estratégia Saúde da Família em Sobral, CE. In: Moyses ST, Kriger L, Moyses SJ: Saúde Bucal das famílias: Trabalhando com evidências. São Paulo: Artes Médicas; 2008. p. 142-153.

30. Trad LAB, Bastos ACS. O impacto sócio-cultural do Programa de Saúde da Família (PSF): uma proposta de avaliação. Cad Saude Publica 1998; 14(2):429-435.

31. Brasil. Ministério da Saúde (MS). Secretaria de Gestão Estratégica e Participativa. Departamento de Apoio à Gestão Participativa. Caderno de educação popular e saúde. Brasília: MS; 2007.

32. Costa ICC. O paradigma da promoção da saúde e sua interface com a saúde bucal. In: Ferreira MAF, Roncalli AG, Lima KC. Saúde bucal coletiva: conhecer para atuar. Natal: EDUFRN; 2004. p.163-173.

33. Valença AMG. A educação em saúde na formação do cirurgião-dentista: de necessidade à prática participativa. Niterói: EDUFF; 1998

34. Emmi DT, Barroso RFF. Avaliação das ações de saúde bucal no Programa Saúde da Família no distrito de Mosqueiro, Pará. Cien Saude Colet 2008; 13(1):35-41.

Artigo apresentado em 29/09/2011

Aprovado em 03/12/2011

Versão final aprovada em 05/01/2012 